\title{
A Novel Robust Scaling for EDM Calibration Baselines using Monte Carlo Study
}

\author{
Ramazan CUNEYT ERENOGLU
}

\begin{abstract}
The terrestrial measurements using Electronic Distance Measurement (EDM) have been widely done for different applications such as deformation monitoring and establishing geodetic networks. The calibration of the EDMs reflects the quality of the estimated parameters. In geodesy, least squares principle is mainly used for estimating parameters. The least square estimation is adversely affected by the systematic and non-systematic errors resulting in bias for the estimated parameters. In this study, to compare efficacy of different robust methods, the Monte Carlo simulation is applied to the EDM calibration as well as real experiments. The parameters without errors are obtained as a result of the used methodology. The methods given in this study are basically based on iteratively reweighted least squares and can be used for both parameter estimation and outlier diagnostics. This is of particular importance for calibrations of electromagnetic distance measurements using the Monte Carlo simulation and the measured test baselines. The results showed that one of the advantages of the used methodology is the improvement of the reliability of the estimated calibration parameters.
\end{abstract}

Keywords: calibration; electronic distance meter; least squares estimation; Monte Carlo simulation; reliability; robust

\section{INTRODUCTION}

Spatial distances are surveyed using different measurement techniques such as electronic, optical and direct methods. Electronic Distance Measurement (EDM) is often used to determine distances by the developed electronic equipment phase difference, pulse, Doppler methods and interferometry [1]. Currently, EDMs basically employed the phase different detection using infrared or light waves that are classified as electro-optic instruments. Microwave instruments are based on radio waves.

The errors disturbing an EDM instrument are internal errors, i.e. zero error, scale error, cyclic error, phase measurement error, and external errors are commonly due to atmospheric refraction [2]. The EDM calibration baselines have to be scaled to determine the scale error, which is formed by changing frequency of proportional part of the scale in time in the electromagnetic distance measurements. On the other hand, true values of certain distances obtained by dividing the whole control baseline and the distances, which are formed as combinations of these distances, have to be known. Calibration of electromagnetic distance measurements and design of EDM calibration baselines were studied by many scientists [1-5].

There are some works for modelling instrumental biases $[2,6,7,8]$. These studies focused on estimating the calibration parameters of Electronic Distance Meter (EDM) by Least Square Estimation (LSE).

Noise level can be handled by fitting a model to data since the real data are contaminated by different reasons. The LSE approach that is a common form of regression analysis can efficiently be applied to normally distributed random errors in EDM calibration [2, 7]. Since LSE approach is extremely sensitive to outliers, several robust methods developed in recent decades were successfully applied to data which includes outliers [9-12]. The robust methods were applied to determine calibration parameters of EDM using just a number of dataset first time [13]. However, it could not provide the efficacy of the robust estimators or LSE in identifying calibration parameters.
In this study, commonly used EDM calibration process is modified using robust estimators in order to estimate calibration parameters and to localize biases. Here, to enable the comparison among the different robust estimators and LSE in various situations, measures of performance are used. One such global performance measure for the robust estimators is to use the Monte Carlo simulation as well as the real experiments. Moreover, it is investigated to determine which of these techniques is best suited for surveying applications.

\section{ERROR SOURCES FOR EDM}

The error sources are classified as internal and external errors. The internal errors for EDMs can be regarded as zero error, scale error, cyclic error and so on. These can occur due to some deficiencies on instruments and reflectors. On the other hand, the external errors are caused by atmospheric refraction.

Zero (additive) error that occurs observing the distance between two marks by EDM is a constant systematic error [13]. This error can be caused by the internal electronic or optical deficiencies of the instruments or improperly centering of the instrument and reflector $[2,14]$.

Scale error is an increasable systematic error. In the generation stage of the modulated signal, there can be deficiencies in electronic oscillator unit because of ageing or temperature changes resulting in scale factor for EDM. It means that discrepancies between design frequency and modulated frequency result in change of wavelength for the instrument. Atmospheric changes, such as atmospheric pressure and temperature, affect the velocity of the electromagnetic signal [13].

The equation for the wave:

$v=f \times \lambda$

where $v$ is the velocity, $f$ is the frequency, $\lambda$ is the wavelength. The frequency of the wave is constant. It can be represented by the scale error as parts per million (ppm). 
Cyclic error is caused by the non-linearity in amplitude modulation of the phase and carrier wave measurement. This cyclic error changes across the modulated wavelength. This error is normally small for an EDM instrument in good adjustment. But, its presence must be checked as an indication of the adjustment of the EDM instrument. Cyclic error is usually a periodic, sinusoidal, in nature with a wavelength equal to the unit length of the EDM. Cyclic error can be regarded as systematic error. The unit length is the scale on which the EDM measures the distance. Unit length is as great as one half of the modulation wavelength $[13,15]$.

Other errors can be regarded as pointing errors that take place of misalignment of the telescope to the target. This is resulting in weak signal strength and makes it difficult to take observation. Telescope of EDM should be directed to target until maximum signal is gained.

Even though there are many error sources to be addressed, the evaluation of the zero and the scale errors is the aim of the calibration process [2]. The zero and scale errors can simultaneously be estimated using the baselines with different distances between the different points.

\section{OUTLIER CONCEPT}

Outlier analysis is a very important concept in all kinds of scientific studies [16]. There are several techniques to get rid of the outliers in the data set. If the number of the sample increased, the observations are mentioned as normally distributed according to the central limit theorem. Deviations from the normal distribution can be analysed by using confidence interval. Confidence interval defines reliability of the estimated distribution. A confidence interval can be changed using confidence level that is expressed as a percentage. The end points of the confidence interval managed by confidence level can be mentioned as confidence limits. The measurements exceed the limit value regarding as outliers are discarded from the data set.

In statistics, one pays more attention to outliers that render the estimation of unknowns meaningless. For example, the least squares estimator has a breakdown point of $0 \%$, because the presence of only one outlier affects the LS estimator to produce arbitrary biased solutions. There are several robust estimators studied by the scientific community.

The robust M-estimation, a generalized form of maximum likelihood estimation (MLE), was presented by Huber [17]. Iteratively reweighted LS estimation can be used to solve non-linear normal equation system of the M-estimation [18]. The M-estimation of Huber, the Mestimation of Hampel, the M-estimation of Andrews, the Danish method, the $L_{1}$-norm and the IGGIII scheme were used [9, 10, 19-23]. Even though the LMS method was assumed as the most robust method by [11], it was shown in [24] that this method failed with a single influential outlier.

Outliers are assumed as coming from different set of observation group in [16]:

$$
F(x)=(1-\varepsilon) F_{0}(x)+\varepsilon H(x)
$$

where $F(x)$ is the distribution function of observation set, $H(x)$ is the distribution function of outliers, $F_{0}(x)$ is the distribution function of good observations and $\varepsilon$ is the breakdown degree. If $F_{0}(x)$ and $H(x)$ are normal distributed functions as $F_{0}(x) \sim N\left(\mu_{1}, \sigma_{1}^{2}\right)$ and $H(x) \sim$ $N\left(\mu_{2}, \sigma_{2}^{2}\right)$.

Distribution function of observation set can be given as follows:

$$
F(x)=(1-\varepsilon) N\left(\mu_{1}, \sigma_{1}^{2}\right)+\varepsilon N\left(\mu_{2}, \sigma_{2}^{2}\right)
$$

where $\mu$ is mean value of normal distribution and $\sigma^{2}$ is the variance of the distribution. As it can be seen from Eq. (2), outliers come from separate distribution [25]. Observations with $N\left(\mu, \sigma^{2}\right)$ that comes from the same distribution can be mentioned as good observations. Outliers are assumed in the interval of $\left(-\infty, \mu-z_{1-\alpha / 2}\right)$ and $\left(\mu+z_{1-\alpha / 2},+\infty\right)$ where $\alpha$ is the significance level, $z_{1-\alpha / 2}$ is the corresponding critical value.

The observations are taken into account as deterministic and stochastic components:

$l_{i}=l_{d_{i}}+l_{s_{i}}$,

where $l_{i}$ is the observation, $l_{d_{i}}$ is the deterministic part of the observation and $l_{s_{i}}$ is the stochastic part of the observation. Outliers can be regarded as

$\bar{l}_{i}=l_{d_{i}}+\delta l_{i}$,

where $\bar{l}_{i}$ is the observation which includes the outliers and $\delta l_{i}$ is the outlier. Outliers can take values between the given intervals as $-\infty<\delta l_{i}<-s_{d}$ or $s_{d}<\delta l_{i}<\infty$ where $s_{d}$ is the limit value that is chosen as $3 \sigma$.

Huber has been a pioneer [17] to robust statistics and many investigators such as $[10,20]$ contributed to improve this statistics. They aimed to estimate unknown parameters and standard deviations discarding the disturbing effects of the outliers in such quantities. The benefit of the robust estimation is to reduce or even eliminate the effects of outliers on the estimated parameters [26].

Robust estimation is not sensitive to small deviations from model assumptions. It limits the effects of the outliers in the observations. The outliers do not affect all the residual that only magnifies related residual [27]. Robust M-estimation uses iterative re-weighting LS during the solution of the unknown. In this process, the a priori weight of the observations is converted to pseudoweight in the iteration step. If any observation includes outlier, pseudo-weight will get smaller even rapidly converging to zero. This feature provides recognition of the outliers in the observations.

M-estimator which generalizes Maximum Likelihood estimator was introduced for location parameter of a distribution in [17] as follows:

$\boldsymbol{M}=\sum_{i=1}^{n} \rho\left(\sum_{i=1}^{n} a_{i j} x_{j}-l_{j}\right)=\sum_{i=1}^{n} \rho\left(v_{i}\right)=\min$

where $\rho\left(v_{i}\right)$ is the lost function. The derivation of $\rho\left(v_{i}\right)$ with respect to $v_{i}$ gives influence function as 


$$
\frac{\partial \rho\left(v_{i}\right)}{\partial v_{i}}=\psi\left(v_{i}\right)
$$

Derivation of $\boldsymbol{M}$ with respect to the unknowns assuming $v_{i}$ as the function of the unknowns,

$$
\frac{\partial \boldsymbol{M}}{\partial x_{j}}=\sum_{i=1}^{n} \frac{\partial \rho\left(v_{i}\right)}{\partial v_{i}} \frac{\partial v_{i}}{\partial x_{j}}=\sum_{i=1}^{n} \psi\left(v_{i}\right) \frac{\partial v_{i}}{\partial x_{j}}=0
$$

or

$$
\sum_{i=1}^{n} \psi\left(v_{i}\right) a_{i j}=0
$$

with matrix notation

$$
A^{\mathrm{T}} \psi(v)=A^{\mathrm{T}} \psi(A \hat{x}-l)=0
$$

Eq. (9) is usually not linear unless residuals are normally distributed. If the residuals are normally distributed the solution is identical with LS [27].

If Eq. (9) is multiplied and divided with $(A \hat{x}-l)$,

$$
W=W(v)=\frac{\psi\left(v_{i}\right)}{v}=\frac{\psi(A \hat{x}-l)}{A \hat{x}-l}
$$

is obtained. After transformation, the following equation is attained as:

$$
A^{\mathrm{T}} W(v)=A^{\mathrm{T}} W(A \hat{x}-l)=0
$$

This equation is similar to the LS normal equation. After this equation arranged,

$$
\hat{x}=\left(A^{\mathrm{T}} W A\right)^{+} A^{\mathrm{T}} W l
$$

A number of robust methods have been proposed, which include the M-, R-, and L-estimators, the $L_{1}$-norm, the least median of squares (LMS) and the signconstrained robust least squares [9, 10, 11, 22, 24]. If the variance-covariance (VCV) matrix of the observations is given, the M-estimates have been extended to include correlated observations [28, 29]. Robust methods have also been applied to geodetic networks [26, 30-38].

\section{MATHEMATICAL MODEL FOR EDM}

In the mathematical model for EDM calibration, the observed baselines and zero error will be estimated using the conventional least squares and robust methods. The basic equation for adjustment can be given as follows:

$$
l^{c}=l+z_{0}
$$

where $z_{0}$ is the zero error, $l$ is the measured distance and $l^{c}$ is the corrected distance. Zero error can be defined by using observed baselines. The general linear or linearized observational parametric model can be given as:
$l+v=A x$

where $\boldsymbol{A}$ is the design matrix; $x$ is the vector of unknown parameters; $l$ is the vector of observations; and $v$ is the vector of residuals. The least squares solution $\hat{x}$ of Eq. (15) is as follows [39]:

$\hat{x}=\left(A^{\mathrm{T}} P A\right)^{-1} A^{\mathrm{T}} P l=N^{-1} A^{\mathrm{T}} P l, C_{\hat{x}}=\sigma_{0}^{2}\left(A^{\mathrm{T}} P A\right)^{-1}$

where $\boldsymbol{P}$ is the weight matrix; $\boldsymbol{C}_{\hat{x}}$ covariance matrix of parameters; $\boldsymbol{N}$ is the normal equation matrix and $\sigma_{0}^{2}$ is the a priori variance factor. The adjustment model can be given as:

$l_{1}+v_{1}=x_{1}-z_{0}$

$l_{2}+v_{2}=x_{1}+x_{2}-z_{0}$

...

$l_{m}+v_{m}=x_{1}+x_{2}+\ldots \ldots+x_{m}-z_{0}$

Calibrations of a higher precision can be achieved by occupying all pillars and measuring all combinations of distances on a baseline. The baselines available for calibration and standardization have specially constructed pillars for the stations. The main reasons are:

- Forced centring is essential to eliminate setting-up errors,

- The speed and ease of the calibration procedure is enhanced,

- A precision EDM is able to be used to its full capacity and

- Constant instrument heights are obtained.

Calibration techniques assume that there is no pillar movement between the time when the baseline is certified and when the user calibrates the EDM. Baseline stability is closely monitored to ensure that calibrations can be performed to the required precision.

\section{EXPERIMENTAL RESULTS, ANALYSIS AND DISCUSSION}

Tests were performed to evaluate the efficiency of the robust methods in comparison with a conventional LSE. However, prior to the testing, the MATLABR2016a v.9.0 script was enhanced to compute the parameters to be estimated [40]. Before beginning the experiments, a check was done to grasp how to run the system. This was done to confirm that measuring baselines were in common between the conventional LSE method and the robust method. In the conventional method, parameter estimation is performed using the LSE method. For the robust methods, on the other hand, the iteratively reweighted least squares approach is replaced by LSE in the parameter estimation. All the parameters, i.e. adjusted sub-baselines and the zero error, could be obtained after the measuring procedure through the user interface in the MATLAB script. Therefore, the result is ready almost immediately after all the points are measured. The efficiency of the robust methods for parameter estimation has been tested on different baselines for special cases. The results of baseline solutions are the outcome of a 
simulation study and real data measured in Terzioglu Campus, Canakkale.

\subsection{Simulation}

EDM can be calibrated by measuring a combination of distances on a baseline. An important feature of baseline design is to enable the determination of all instrument errors to an appropriate level of precision. One objective of the design is to ensure that the additive constant and scale error are determined independent of any cyclic error contributions. However it is not possible to solve for a scale error unless the inter-pillar distances for the baseline are known. So, in the first example, the basic baseline between $\mathrm{A}$ and $\mathrm{E}$ points, totally 400 meters, has been divided into four equal parts. It is considered that the length of each part is 100 meters. These baselines are given in Fig. 1. To investigate the quality of calibration of the instruments and estimated baselines by the various methods, some special samples are required, for example Monte Carlo simulation. Therefore, various examples will be first shown in a simulation study that the calibration process using the LSE is comparable to that of other robust methods. The key advantage of the Monte Carlo simulation is that the optimal quality criteria are guaranteed between estimated parameters and their known values.

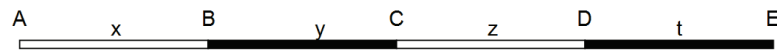

Figure 1 Sub-divisions of simulated baseline

Let us take a simulated baseline which consists of four equal parts given in Fig. 1. Each part is 100 meters. Various combination scenarios based on this baseline are considered, each being a four or component mixture of normal components. Tab. 1 shows these scenarios and the lengths of the baselines. Let the sample $o$ be formed by including the baselines. A contaminated sample $\bar{o}$ is obtained by adding some kinds of errors to the original baselines $o$. After applying LSE method and robust estimator to the contaminated sample $\overline{\mathbf{0}}$, the baselines and the zero error will be computed.

Table 1 Various measurement scenarios

\begin{tabular}{|c|c|c|c|c|c|}
\hline Point \# & A & B & C & D & E \\
\hline A & & $100 \mathrm{~m}$ & $200 \mathrm{~m}$ & $300 \mathrm{~m}$ & $400 \mathrm{~m}$ \\
\hline B & & & $100 \mathrm{~m}$ & $200 \mathrm{~m}$ & $300 \mathrm{~m}$ \\
\hline C & & & & $100 \mathrm{~m}$ & $200 \mathrm{~m}$ \\
\hline D & & & & & $100 \mathrm{~m}$ \\
\hline E & & & & & \\
\hline
\end{tabular}

For the baselines, the observations, such as baseline measurements $o$ are computed from Tab. 1. They are not affected by random errors. First, the random errors for these baselines have been simulated by randn.m function of the MATLAB software. By using the random number generator, the random errors are generated from the normal distribution, i.e. $e \sim N\left(0, \sigma^{2}\right)$ where $\sigma=3 \mathrm{~mm}+2$ ppm.

To obtain good distance measurements $o_{2 j}{ }^{\prime}$, random errors of $e_{o 2 j}$ are added to o as follows:

$\bar{o}_{j}=o_{j}+e_{j}$ where $n$ is the number of the observation. As mentioned before, primary sources of these errors are EDM instrument centering errors, prism centering errors, atmospheric conditions etc. The baselines are also intentionally erroneous by a constant systematic error, i.e. zero error, $0.009 \mathrm{~m}$. Moreover, in order to contaminate the sample, a baseline is randomly chosen for each sample and we have replaced the random error by a blunder, i.e. gross error, whose magnitude is $0.05 \mathrm{~m}$. Finally, we have a hundred of different contaminated samples $\bar{O}$ which includes random errors, zero error and also gross errors.

The random errors must be computed according to the rules of errors propagation to determine their effect on parameter estimation for surveying measurements. Moreover, the disturbing effect of the outliers must be reduced on parameter estimation. To do it, robust methods are used as a statistical tool in order to reduce or remove the effect of outlying data. The conventional LSE estimator and five robust methods are applied to the simulated samples. The robust methods used in this study are Andrews, Danish, Hampel, $L_{1^{-}}$Norm and IGG-3 [10, $19,20,21,22,29]$. The aim is to estimate each subbaseline of 100 meters and also zero error of the instrument. In this study, the tuning constant, c is chosen as 1.5 for robust methods except for Hampel's method. For Hampel's method the values of $\mathrm{a}, \mathrm{b}$ and $\mathrm{c}$ are taken as 0.8, 1.6 and 3.2. All the results attained have been calculated using a computer program in MATLAB (v 6.5) in which subroutine rob.m and linprog.m. The weighting functions of the robust methods will be given in the appendix. Note that in the design matrix $A$ given in Eq. (18), the unknown parameters to be estimated are $x, y, z$ and $t$ baselines and also $\mathrm{z}_{0}$ zero error for the EDM instrument. In this section, we make use of solutions based on robust approach. Comparing the results to the ones from the conventional LSE, a hundred different samples are firstly analyzed simulated by the Monte Carlo method. The results of the adjustment for estimating the zero error and the unknown baselines using LSE, Andrews, Danish, Hampel, $L_{1}$ - Norm and IGG-3 methods are computed.

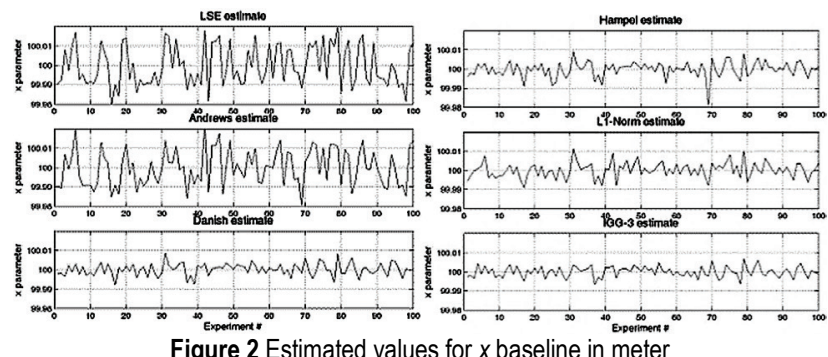

The procedure was applied as described above to compute four sub-baselines, i.e. $x, y, z$ and $t$ given in Fig. 1 , for a hundred separate experiments. For reasons of comparison, the results of the methods are presented together. Fig. 2 shows the resulting $\mathrm{x}$ unknown for the experiments. As seen from here, the largest differences compared to the rigorous solution are obtained for the LSE. Even one error can badly affect the estimates of LSE. Therefore, the errors must be analyzed and eliminated on the final solution. To do it, we use the robust approach to the same samples. When comparing 
the result from the robust methods from Fig. 2, all the robust methods provide smaller differences than the ones from LSE, except for Andrews' estimation. Especially, Danish, $L_{1^{-}}$Norm and IGG-3 give the best solutions for the $\mathrm{x}$ baseline. They have minimal differences from the rigorous solution.

Here, similar results are obtained for the estimation of the other baselines. Figs. 3, 4 and 5 show the solutions of $y, z$ and $t$ baselines, respectively. The largest differences in amount are the solutions for conventional LSE. It is clear that LSE is very sensitive against the errors. On the other hand, the robust methods successfully eliminated disturbing effects of this kind of errors on the final solution.

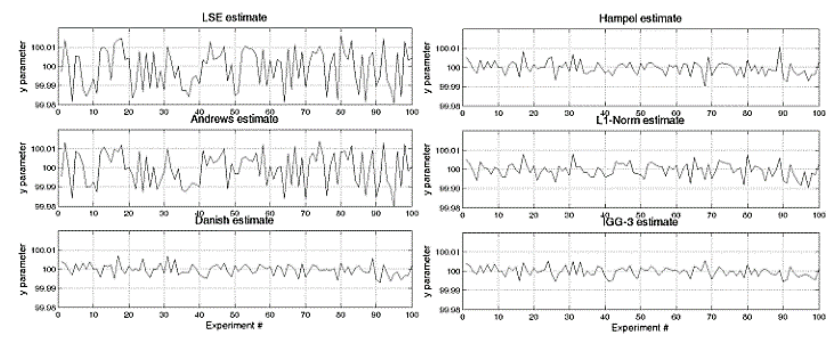

Figure 3 Estimated values for $y$ baseline in meter

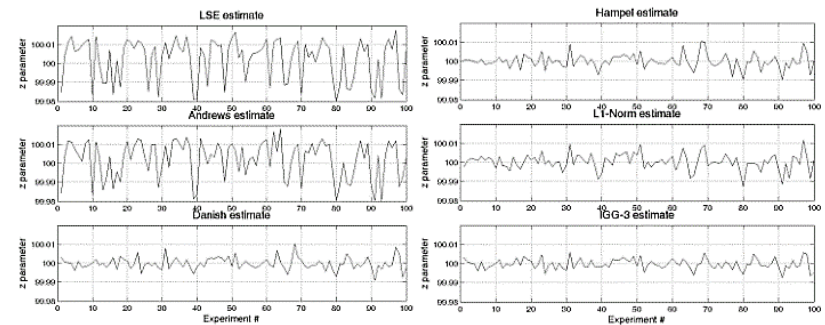

Figure 4 Estimated values for $z$ baseline in meter

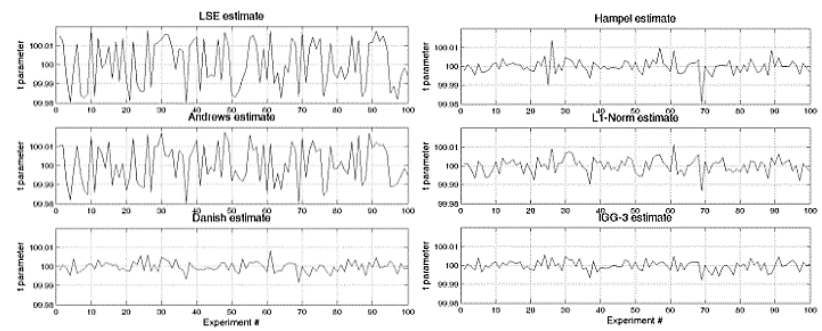

Figure 5 Estimated values for $t$ baseline in meter

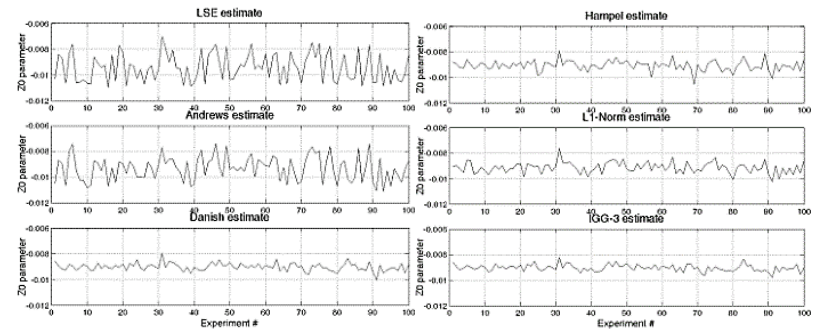

Figure 6 Estimated values for zero error $\left(z_{0}\right)$ baseline in meter

Furthermore, we concentrate on the estimation of the $z_{0}$ zero error for the EDM instrument. Note that $0.009 \mathrm{~m}$ of the zero error is intentionally simulated and added to all the baselines. Fig. 6 shows the solution of $z_{0}$ error. Danish method gives the most successful estimation for $z_{0}$. In addition, LSE has the largest differences compared to the rigorous solution for a hundred samples.

\subsection{Real Baselines}

In the practical applications, calibrations of a higher precision can be achieved by occupying all pillars and measuring all combinations of distances on a baseline. For this purpose, the cadastral surveying system finally used total station surveying technology to conduct precise observations of prism reflectors. The baselines available in Terzioglu Campus, Canakkale, have specially established the concrete benchmarks for the stations. Fig. 7 illustrates these benchmarks as well as the baselines and some buildings around. As seen from Fig. 7, there are 6 pillars. In the conventional method, generally, two people can form a survey crew. One person typically handles the reflector prism. The other one of the crew is located at the instrument point, actually operates the instrument and handles the field book. Real observations have been carried out by the Kolida KTS-442 RLC total station. Note that Kolida KTS-442RLC with the following technical performance: precision angular measurements 1 1", $5000 \mathrm{~m}$ distance measurements reflector $2 \mathrm{~mm}+2$ ppm, typical measurement times $0.5 \mathrm{~s}$. All the combinations of distances (sub-baselines) have been measured independently 10 times.

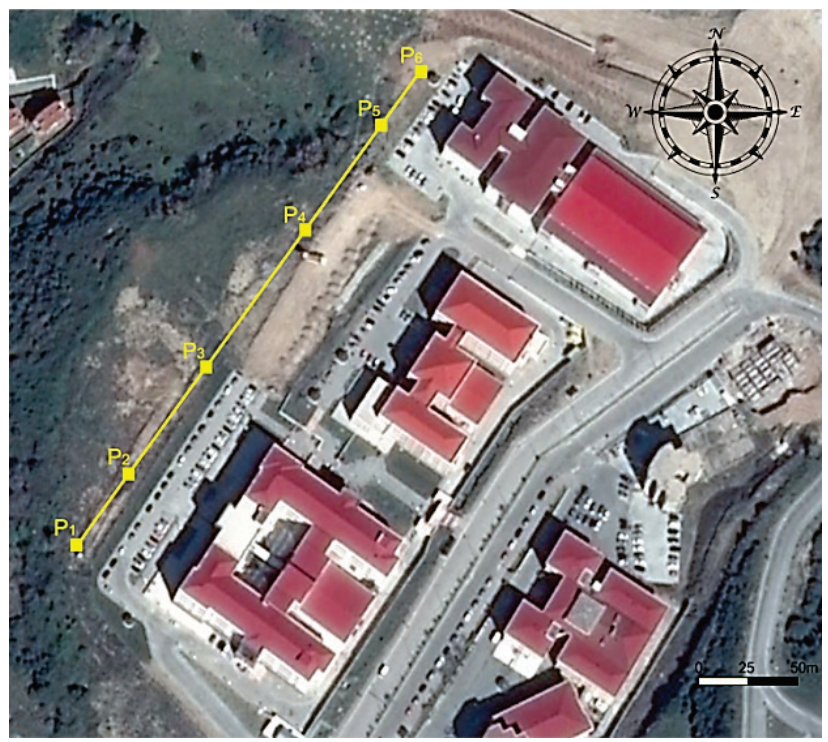

Figure 7 The benchmarks and baselines in Terzioglu Campus

Table 2 The measured baselines (in meter)

\begin{tabular}{|c|c|c|c|c|c|c|}
\hline $\begin{array}{c}\text { Point } \\
\#\end{array}$ & $\mathrm{P}_{1}$ & $\mathrm{P}_{2}$ & $\mathrm{P}_{3}$ & $\mathrm{P}_{4}$ & $\mathrm{P}_{5}$ & $\mathrm{P}_{6}$ \\
\hline $\mathrm{P}_{1}$ & & 41.1529 & 94.4551 & 170.0693 & 224.6190 & 256.1395 \\
\hline $\mathrm{P}_{2}$ & 41.1523 & & 53.2766 & 128.8881 & 183.4368 & 214.9542 \\
\hline $\mathrm{P}_{3}$ & 94.4562 & 53.2770 & & 75.5871 & 130.1351 & 161.6556 \\
\hline $\mathrm{P}_{4}$ & 170.0657 & 128.8858 & 75.5861 & & 54.5262 & 86.0441 \\
\hline $\mathrm{P}_{5}$ & 224.6178 & 183.4353 & 130.1358 & 54.5266 & & 31.4931 \\
\hline $\mathrm{P}_{6}$ & 256.1382 & 214.9531 & 161.6548 & 86.0443 & 31.4938 & हn \\
\hline
\end{tabular}

The mean values of the measured distances between the pillars given in Tab. 2 show that the number of observations is 30 , the number of unknown parameters is 6 , the degree of freedom is 24 , and the accuracy of these observations is $2 \mathrm{~mm}+2 \mathrm{ppm}$. We formed the design matrix $A$ given in Eq. (18), and the unknown parameters are $\overline{\mathrm{P}_{1} \mathrm{P}_{2}}, \overline{\mathrm{P}_{2} \mathrm{P}_{3}}, \overline{\mathrm{P}_{3} \mathrm{P}_{4}}, \overline{\mathrm{P}_{4} \mathrm{P}_{5}}, \overline{\mathrm{P}_{5} \mathrm{P}_{6}}$ and also $z_{0}$ zero error for Kolida KTS-442 RLC used. The results of adjustment for 
estimating these unknowns using LSE and robust approaches are obtained.

The first way is to use the linear model (15) by LSE method and estimate the solution vector for baselines as:

$\hat{x}=\left[\begin{array}{lllll}41.1536 & 53.2778 & 75.5859 & 54.5262 & 31.4929\end{array}\right]_{(\mathrm{m})}^{\mathrm{T}}$

$\hat{\mathrm{z}}_{0}$ and $\sigma_{\hat{\mathrm{z}}_{0}}$ are computed as $-1.2 \mathrm{~mm}$ and $2.4104 \mathrm{~mm}$, respectively.

Furthermore, we computed the same unknown using Danish, $L_{1}$-Norm and IGG-3 methods. The results of baselines from Danish method:

$\hat{x}=\left[\begin{array}{lllll}41.1513 & 53.2769 & 75.5860 & 54.5276 & 31.4923\end{array}\right]_{(\mathrm{m})}^{\mathrm{T}}$

$\hat{\mathrm{z}}_{0}$ and $\sigma_{\hat{\mathrm{z}}_{0}}$ are computed as $-0.8 \mathrm{~mm}$ and $1.8428 \mathrm{~mm}$, respectively.

The baselines from $L_{1}$-Norm method are as: $\hat{x}=\left[\begin{array}{lllll}41.1534 & 53.2768 & 75.5862 & 54.5240 & 31.4930\end{array}\right]_{(\mathrm{m})}^{\mathrm{T}}$ $\hat{\mathrm{z}}_{0}$ and $\sigma_{\hat{\mathrm{z}}_{0}}$ are computed as $-0.6 \mathrm{~mm}$ and $1.6437 \mathrm{~mm}$, respectively.

The adjusted baselines from IGG-3 scheme are as follows:

$$
\hat{x}=\left[\begin{array}{lllll}
41.1519 & 53.2772 & 75.5876 & 54.5279 & 31.4937
\end{array}\right]_{(\mathrm{m})}^{\mathrm{T}}
$$

$\hat{\mathrm{z}}_{0}$ and $\sigma_{\hat{\mathrm{z}}_{0}}$ are computed as $-0.7 \mathrm{~mm}$ and $1.6145 \mathrm{~mm}$, respectively.

From the above results, the simple derived formula based on the robust approach produces better solutions for estimating baseline distances. In the previous studies, the conventional least square approach was only used for estimating calibration parameters of $\operatorname{EDM}[2,7,8]$. However, in this study, in terms of the reliability and accuracy of criterion-related validity, five robust approaches are used to estimate EDM calibration as well as the lest squares when comparing with similar studies. Here, the standard deviations for the zero error obtained shows that using the robust approach more quality estimation is performed than the least squares. Thus, the unbiased parameters are obtained as a result of above methodology. So, the iteratively reweighted least squares should be substituted into the parametric model for estimating.

\section{CONCLUSION}

In this study, to perform more reliable calibration of EDM, the iteratively reweighted least squares are adopted against various types of errors. For evaluating their efficacy, 5 robust approaches and conventional least squares are compared for EDM calibration. The basic formula is very effective for more reliable solutions in EDM instruments. The efficiency and validity of the suggested robust formula were tested and validated successfully. Results show that the robust approaches provided more reliable results for estimating the calibration parameters comparing to the least squares. In terms of more quality estimation, the robust approaches performed better the standard deviations than the least squares in estimating of the zero error. Furthermore, the estimation of baselines provided more accurately against the outlying data. Moreover, the evaluation of the zero error as the systematic error for EDM instruments is performed more accurately. As a results, there might be some errors/outliers for EDM calibration. In such a case, practitioners should adopt this scheme to the precision of their measurements, for precise work.

\section{Acknowledgement}

The author would like to thank the editor and two anonymous reviewers for their valuable comments.

\section{REFERENCES}

[1] Rüeger, J. M. (1976). Problems in the Joint Determination of Cyclic Error and Additive Constant of Electro-optical Distance Meter. Allgem Vermessungs, 8, 338-344.

[2] Amiri-Simkooei, A. R. (2003). Least-Squares Formula for Zero Error $\left(z_{0}\right)$ of Electromagnetic Distance Measuring Instrument. Journal of surveying Engineering, 129(4), 136140. https://doi.org/10.1061/(ASCE)0733-9453(2003)129:4(136)

[3] Aeschlimann, H. \& Stocker, R. (1974). Instrumental Errors in Electro-optical Distance Measurements, Paper 505.4, $14^{\text {th }}$ FIG Congress, Washington DC. https://doi.org/10.1080/00050326.1978.10441502

[4] Sprent, A. \& Zwart, P. (1978). EDM Calibration - A Scenario. Aust. Surv., 29, 157-169.

[5] Spent, A. (1980). EDM Calibration in Tasmania. Aust. Surv., 30, 213-227. https://doi.org/10.1080/00050326.1980.10440964

[6] Hhalil, R. (2005). Compact Testing of EDM Instrument. GIM International, 18, 4-5.

[7] İnal, C., Şanlıŏlu, İ. \& Yiğit, C. Ö. (2008). Scaling of EDM Calibration Baselines by GPS and Controlling of EDM Parameters. Survey Review, 40(309), 304-312. https://doi.org/10.1179/003962608X325376

[8] Becerra, G. E. V., Bennett, R. A., Chávez, M. C., Soto, M. E. T. \& Gaxiola-Camacho, J. R. (2015). Short Baseline Calibration using GPS and EDM Observations. Geofisica Internacional, 54(3), 255-266. https://doi.org/10.1016/j.gi.2015.04.017

[9] Huber, P. J. (1981). Robust Statistics. John Wiley and Sons, New York. https://doi.org/10.1002/0471725250

[10] Hampel, F., Ronchetti, E., Rousseeuw, P. J. \& Stahel, W. (1986). Robust Statistics: The Approach based on Influence Functions. John Wiley and Sons, New York.

[11] Rousseeuw, P. J. \& Leroy, A. M. (1987). Robust Regression and Outlier Detection. John Wiley and Sons, New York. https://doi.org/10.1002/0471725382

[12] Shevlyakov, G. L. \& Vilchevsk, N. O. (2001). Robustness in Data Analysis: Criteria and Methods. VSP International Science Publishers, Utrecht. https://doi.org/10.1515/9783110936001

[13] Erenoglu, R. C. (2012). A Robust Calibration Scheme for Electromagnetic Distance Measurements. Proceedings of International Symposium on Modern Technologies, Education and Professional Practice in Geodesy and Related Fields / Sofia, 58-67.

[14] Cooper, M. A. R. (1987). Control Surveyings in Civil Engineering. First published in Great Britain by Collins Professional and Technical Books. William Collins Sons and Co. Ltd.

[15] Rüeger, J. M. (1990). Introduction to Electronic Distance Measurement. Thir Totall Revised Edition, Monograph No. 7. School of Surveying, University of New South Wales, Australia. https://doi.org/10.1007/978-3-642-97196-9

[16] Barnett, V. \& Lewis, T. (1994). Outliers in Statistical Data. $3^{\text {rd }}$ ed. John Wiley and Sons, New York. 
[17] Huber, P. J. (1964). Robust Estimation of a Location Parameter. Ann Math Stat, 35, 73-101. https://doi.org/10.1214/aoms/1177703732

[18] Koch, K. R. (1999). Parameter Estimation and Hypothesis Testing in Linear Models. $2^{\text {nd }}$ ed. Springer, Berlin Heidelberg New York. https://doi.org/10.1007/978-3-662-03976-2

[19] Andrews, D. (1974). A Robust Method for Multiple Linear Regression. Technometrics, 16, 523-531. https://doi.org/10.1080/00401706.1974.10489233

[20] Barrodale, I. \& Roberts, F. D. K. (1974). Solution of an Over Determined System of Equations in $\mathrm{L}_{1}$-norm. Communications ACM, 17, 319-320. https://doi.org/10.1145/355616.361024

[21] Krarup, T., Juhl, J. \& Kubik, K. (1980). Götterdaemmerung over Least Squares Adjustment. Proceedings of the $14^{\text {th }}$ Congress of Int. Soc. Photgr. / Hamburg.

[22] Koch, K. R. (1996). Robuste Parameterschaetzung. Allg Vermessungs-Nachr, 103, 1-18.

[23] Yang, Y. (1999). Robust Estimation of Geodetic Datum Transformation. Journal of Geodesy, 73, 268-274. https://doi.org/10.1007/s001900050243

[24] Xu, P. L. (2005). Sign-Constrained Robust Least Squares, Subjective Breakdown Point and the Effect of Weights of Observations on Robustness. Journal of Geodesy, 79, 146159. https://doi.org/10.1007/s00190-005-0454-1

[25] Kubik, K. \& Wang, Y. (1991). Comparison of Different Principles for Outlier Detection. Australian Journal of Geodesy, Photogrammetry and Surveying, 54, 67-80.

[26] Gao, Y., Krakiwsky, E. J. \& Czompo, J. (1992). Robust Testing Procedure for Detection of Multiple Blunders. Journal of Surveying Engineering, 118(1), 11-23. https://doi.org/10.1061/(ASCE)0733-9453(1992)118:1(11)

[27] Caspary, W. F. \& Borutta, H. (1987). Robust Estimation in Deformation Models. Survey Review, 29(223), 29-45. https://doi.org/10.1179/sre.1987.29.223.29

[28] Xu, P. (1989). On Robust Estimation with Correlated Observations. Bulletin Géodesique, 63, 237-252. https://doi.org/10.1007/BF02520474

[29] Yang, Y. (1994). Robust Estimation for Dependent Observations. Manuscr Geod, 19, 10-17.

[30] Fuchs, H. (1982). Contribution to the Adjustment by Minimizing the Sum of Absolute Residuals. Manuscr Geod, 7, 151-207.

[31] Kampmann, G. (1989). Zur Ausgleichung freier Netze mit der L1 norm-methode. Allg. Vermessungs Nachr, 96, 110118.

[32] Harvey, P. R. (1993). Survey Network Adjustments by the L1 Method. Aust J Geod Photogram Surv, 59, 39-52.

[33] Benning, W. (1995). Vergleich Dreier Lp-Schaetzer zur Fehlersuche in Hybriden Lagenetzen. $Z$ Vermessungswesen, 12, 606-617.

[34] Youcai, H. (1995). On the Design of Estimators with High Breakdown Points for Outlier Identification in Triangulation Networks. Bull. Geo. 69, 292-299. https://doi.org/10.1007/BF00806741

[35] Awange, J. L. \& Aduol, F. W. O. (1999). An Evalution of Some Robust Estimation Techniques in the Estimation of Geodetic Parameters. Survey Review, 35(273), 146-162. https://doi.org/10.1179/sre.1999.35.273.146

[36] Wicki, F. Robuste Schätzverfahren für die Parameterschätzung in Geodätischen Netzen. Institut für Geodäsie und Photogrammetrie an der ETH, Zürich, Mitt. Nr. 67. 1999

[37] Berber, M. \& Hekimoğlu, S. (2001). What is the Reliability of Robust Estimators in Networks? Proceedings of the $1^{\text {st }}$ International Symposium on Robust Statistics and Fuzzy Techniques in Geodesy and GIS / ETH, Zurich, 61-66.

[38] Marchall, J. (2002). L1 Norm Pre-analysis Measures for Geodetic Networks. Journal of Geodesy, 76, 345-352.
[39] Wells, D. E. \& Krakiwsky, E. J. (1971). The Method of Least Squares. Dept. of Surveying Engineering, Univ. of New Brunswick, Fredericton, N. B., Canada.

[40] MATLAB - The Language of Technical Computing MathWorks. 2016. http://www.mathworks.com/help/ matlab/(25.03.2016)

[41] Yang, Y., Song, L. \& Xu, T. (2002). Robust Estimator for Correlated Observations based on Bifactor Equivalent Weights. Journal of Geodesy, 76, 353-358. https://doi.org/10.1007/s00190-002-0256-7

\section{Contact information:}

Ramazan CUNEYT ERENOGLU, Ph.D.

Canakkale Onsekiz Mart University,

Faculty of Engineering,

Department of Geomatics Engineering,

17100, Canakkale, Turkey

E-mail: ceren@comu.edu.tr

\section{Appendix:}

\section{Weight Functions of Robust Methods Used in this Study}

\section{A.1 Huber's M-estimation}

Huber's weight function [9] is as:

$W\left(v_{i}\right)= \begin{cases}1 & \left|v_{i}\right| \leq c \\ \frac{c}{v_{i}} & \left|v_{i}\right|>c\end{cases}$

where $c$ is a constant that can be valued as 1.5. $v_{i}$ is the standardized residuals

\section{A.2 Andrew's M-estimation}

The weight function was proposed as follows [19]:

$$
W\left(v_{i}\right)=\left\{\begin{array}{cl}
\left(\frac{\left|v_{i}\right|}{c}\right)^{-1} \sin \left(\frac{\left|v_{i}\right|}{c}\right) & \left|v_{i}\right| \leq c \pi \\
0 & \left|v_{i}\right|>c \pi
\end{array}\right.
$$

where $c=1.5$.

\section{A.3 Danish Method}

Danish method's weight function is as:

$$
f\left(v_{t}\right)=\left\{\begin{array}{cl}
1 & \left|v_{t}\right|<c \\
\exp \left(\frac{\left|v_{i}\right|}{c}\right) & \left|v_{t}\right| \geq c
\end{array}\right.
$$

where $c=3$. This method is proposed to be used for geodetic computations as standard method in Danish Geodetic Institute [21].

\section{A.4 L1-norm Method}

The $L_{1}$-norm is known for being very robust to outliers. It based on the minimization with a linear programming of a modified simplex method [20]. The 
simplex method is a technique designed to solve linear programming problem as:

$\underline{A} \underline{x}=\underline{b}, \underline{x} \geq 0$

The $L_{1}$-norm has been commonly used for two purposes: the estimation of parameters and outlier detection.

\section{A.5 Hampel's M-estimation}

In the Hampel's M-estimation, the weight function is

$$
W\left(v_{i}\right)=\left\{\begin{array}{cc}
1 & 0<\left|v_{i}\right| \leq a \\
\frac{a}{\left|v_{i}\right|} & a<\left|v_{i}\right| \leq b \\
\frac{a\left(c-\left|v_{i}\right|\right)}{\left|v_{i}\right|(c-b)} & b<\left|v_{i}\right| \leq c \\
0 & \left|v_{i}\right|>c
\end{array}\right.
$$

where $a=1.5, b=3$ and $c=6$ are taken [10].

\section{A.6 IGG-3 Scheme}

For the IGG-3, the weight function is as:

$W\left(v_{i}\right)= \begin{cases}1 & \left|v_{i}^{\prime}\right| \leq c \\ \frac{1}{v_{i}^{\prime}} & \left|v_{i}^{\prime}\right|>c\end{cases}$

where $v_{i}^{\prime}=\frac{v_{i}}{\sigma_{0} \sqrt{q_{v_{i}}}}[41]$. 